\title{
Prevalence, causes and management outcome of intestinal obstruction in Adama Hospital, Ethiopia
}

\author{
Urgessa Soressa', Abebe Mamo ${ }^{2,4^{*}}$, Desta Hiko ${ }^{3}$ and Netsanet Fentahun ${ }^{2}$
}

\begin{abstract}
Background: In Africa, acute intestinal obstruction accounts for a great proportion of morbidity and mortality. Ethiopia is one of the countries where intestinal obstruction is a major cause of morbidity and mortality. This study aims to determine prevalence, causes and management outcome of intestinal obstruction in Adama Hospital in Oromia region, Ethiopia.
\end{abstract}

Method: A hospital based cross-sectional study design was used. Data covering the past three years were collected from hospital medical records of sampled patients. The collected data were checked for any inconsistency, coded and entered into SPSS version 16.0 for data processing and analysis. Descriptive and logistic regression analyses were used. Statistical significance was based on confidence interval (Cl) of $95 \%$ at a $p$-value of $<0.05$.

Result: 262 patients were admitted with intestinal obstruction. The prevalence of intestinal obstruction was $21.8 \%$ and $4.8 \%$ among patients admitted for acute abdomen surgery and total surgical admissions, respectively. The mortality rate was $2.5 \%$ (6 of 262). The most common cause of small bowel obstruction was intussusceptions in 48 patients (30.9\%), followed by small bowel volvulus in 47 patients (30.3 \%). Large bowel obstruction was caused by sigmoid volvulus in 60 patients (69.0\%) followed by colonic tumor in 12 patients (13.8\%). After controlling for possible confounding factors, the major predictors of management outcome of intestinal obstruction were: duration of illness before surgical intervention (adjusted odds ratio $(A O R)=0.49,95 \% \mathrm{Cl}$ : 0.25-0.97); intra-operative findings Viable small bowel volvulus (SBV) (AOR $=0.08,95 \% \mathrm{Cl}: 0.01-0.95$ ) and viable (AOR $=0.17,95 \% \mathrm{Cl}: 0.03-0$. 88)]; completion of intra-operative procedures (bowel resection \& anastomosis (AOR=3.05, $95 \%$ Cl: 1.04-8.94); and length of hospital stay ( $\mathrm{AOR}=0.05,95 \% \mathrm{Cl}$ : 0.01-0.16).

Conclusion: Small bowel obstruction was more prevalent than large bowel obstruction. Intussusceptions and sigmoid volvulus were the leading causes of small and large bowel obstruction. Laparotomy was the most common methods of intestinal obstruction management. Bowel resection and anastomosis was the commonest intra-operative procedure done and is associated with postoperative complications. Wound infection in the affected area should be improved because it is the most common postoperative complication. This can be decreased by appropriate surgical technique and wound care with sterile techniques.

Keywords: Intestinal obstruction, Prevalence, Causes, Management, Hospital, Ethiopia

\footnotetext{
*Correspondence: abema24@yahoo.com; abemam24@gmail.com

${ }^{2}$ Department of Health Education and Behavioral Sciences, Jimma University,

Jimma, Ethiopia

${ }^{4}$ Department of Health Education and Behavioral Sciences, College of Health

Sciences, Jimma University, PO Box 378, Jimma, Ethiopia

Full list of author information is available at the end of the article
} 


\section{Background}

Intestinal obstruction (IO) is defined as obstruction of the passage of the intestine for its contents [1]. It is potentially risky surgical emergency associated with high morbidity and mortality [2]. Emergency operation being defined as those types of surgeries that should be performed by necessity within $24 \mathrm{~h}$ of a patient's admission, or within $24 \mathrm{~h}$ of the development of a specific complication [3]. The research community in both developed and developing countries has investigated this condition [1, 4].

Of all IO, mechanical IO forms an important part of pathologies that necessitate emergency surgical interventions in parts of Asia, including India, Iran and Pakistan $[3,5]$. With certain exceptions, mechanical IO can generally be relieved through conservative treatments like naso-gastric tube insertion, intravenous antibiotics or intravenous fluid resuscitation; unrelieved IO necessitates further exploration. Previous studies revealed that repeat IO will recur in about $12 \%$ of patients after primary conservative treatment, and in between 8 and $32 \%$ of patients after operative management for adhesive bowel obstruction $[2,5]$.

In $80 \%$ of cases, IO occurs in the small bowel, while in $20 \%$ of cases it occurs in the large intestine [1]. There are four cardinal features of IO: colicky abdominal pain, distension, vomiting, and constipation. The presentation of these symptoms is affected by the site and type of obstruction $[2,6]$.

In rural Africa, acute intestinal obstruction accounts for a great proportion of morbidity and mortality [5], and Ethiopia is one of the countries where intestinal obstruction constitutes a major cause of morbidity and mortality [7]. While some studies have been done to assess prevalence and causes of IO in developed countries, the condition remains largely unstudied in the Ethiopia context. With only a few studies conducted in north and central Ethiopia $[8,9]$, there is a lack of research about the prevalence and causes of IO in Ethiopia, particularly in the western and eastern parts of the country. Furthermore, there is no recently published literature that has explored IO in rural and regional hospitals. Thus, this study was conducted to fill this information gap and generate base line information about prevalence, causes and management outcome of IO in rural Ethiopia.

The results of this study will provide epidemiological and clinical information that will serve as an essential input for policy makers to design proper strategies to address IO. Moreover, the study results will serve as references for those who want to undertake research on the prevalence and causes of IO.

\section{Methods}

\section{Study design and period}

Hospital based cross sectional study design was conducted at Adama Hospital Medical College, drawing upon medical records dated January 1, 2011 to December 30, 2013. The data collection period was from February 1-15, 2014.

\section{Study population}

The study population included all patients admitted with diagnosis of IO at Adama Hospital Medical College during the three year period. The following types of patients were included: patients whose IO was managed conservatively without operation; patients who underwent an operation for IO; and patients who died due to a confirmed diagnosis of IO. Patients who had incomplete records (i.e., important information on causes and management outcome variables), or whose records were lost were excluded from further analysis.

\section{Participant selection}

A subsample of data was obtained from all patients admitted to the surgical ward of Adama Hospital Medical College with an IO diagnosis and treated from January 1, 2010 to December 30, 2012. Therefore, 262 patients were admitted for IO during the study period, the data of 242 patients were included for further study.

\section{Data collection methods}

The data were collected by reviewing the registration books using structured checklists. A check list was developed in the English language to collect important information such as age, sex, admission diagnosis, intraoperative findings, intra-operative procedures, duration of presentation, causes of IO, postoperative complications and management outcome. For data collection, two clinical nurses were recruited who were not part of the Adama Hospital Medical College staff. The Principal Investigator provided training for data collectors on how to complete the checklist, as well as the importance of data quality and relevance of the study. Another clinical nurse (a first-degree holder) supervised the data collection activities, ensuring the consistency and completeness of the checklist and providing appropriate support. The Principal Investigator oversaw the daily activities of data collectors and supervisor.

\section{Data collection techniques and measurements}

Patients that were admitted to surgical wards with the diagnosis of IO were initially identified from admission logbooks of surgical wards and operation theaters of the hospital from which the card number of patients was obtained. Using the cards as a reference, patients were 
identified, and relevant information was collected about patients admitted with the diagnosis of IO.

The concepts measured in this study were defined based on a review of relevant literature that has assessed similar key variables as well as earlier studies on managing outcome of IO in health institutions. For the purposes of this study, conservative management includes the management of patients with partial bowel obstruction or recurrent adhesive obstruction; it also includes the management of patients during the early postoperative period with naso-gastric tube NGT suction, intravenous (IV) fluids and frequent clinical reassessment. (The purpose of frequent clinical assessment is to rule out bowel strangulation, which may need operative management.) Operative management includes surgical exploration or operations performed on the abdomen to relieve the causes of obstruction.

A favorable outcome was achieved if patients did not develop either postoperative complication or death after conservative or operative management of IO. If the patient developed one or more postoperative complications (including wound infection, facial dehiscence, anastomotic leakage, developed septic shock, pelvic collection and pneumonia) and/or death this was considered an unfavorable outcome of IO.

Data processing, analysis, interpretation and presentation Data were cleaned, coded and entered into the computer using Epi Data version 3.1, and were subsequently exported to SPSS version 16.0 for further analysis. Results were presented using frequency tables, graphs and percentage. Bivariate logistic regression analysis was done to determine crude statistical associations between independent variables and dependent variables (management outcome of IO). Variables with a $p$-value of less than 0.025 in binary logistic regression analysis were considered as a candidate to be entered into multivariate logistic regression. Multivariable analyses were used to isolate the independent predictor of management outcome of IO. Statistical significance was based on a $p$-value of $<0.05$ with a confidence interval (CI) of $95 \%$.

\section{Data quality management and collection}

Before data collection, the checklists were assessed by principal investigator as well as facilitators and supervisors were trained for two days. To avoid interpersonal variation between data collectors, the same two data collectors were retained throughout the data collection process. Regular daily supervision by the Principal Investigator supported the consistency and completeness of checklists. The completed checklists were checked for their completeness and consistency at every step of data collection. After data collection and before starting data analysis, checklist completeness was rechecked.

\section{Results}

\section{Demographic characteristics}

Over the study period, 5500 patients were admitted to the surgical ward, of which 1200 patients were admitted with diagnoses pertaining to acute abdomen conditions. Two hundred sixty two patients were admitted to the surgical ward with IO of whom data of 242 (92.4 \%) patients were retrieved for further analysis. The minimum age was 1 month and maximum was 85 years with a mean of 32.8 years (standard deviation (SD) \pm 22.6 ) years. The study revealed that 191 (78.9\%), 157 (64.9\%) and $90(36.8 \%)$ of the study participants were males, from rural areas, and farmers, respectively.

\section{Types and prevalence of intestinal obstruction}

The prevalence of IO was $21.8 \%$ among patients admitted with the acute abdomen conditions, and $4.8 \%$ among total surgical admission patients. Out of the 242 patients with IO who underwent further analysis, $64 \%$ had cases of small bowel obstruction and $36.0 \%$ had cases of large bowel obstruction. Of these 242 patients, $13.6 \%$ had a previous history of abdominal operation: in 30 patients (12.4\%) the operation was for adhesion, and in \#\# patients $(31.2 \%)$ the operation was for acute appendicitis (Table 1).

Table 1 Types of intestinal obstruction and its prevalence in Adama hospital medical college, February, 2014

\begin{tabular}{lcl}
\hline Variable & Frequency & Percent \\
\hline Kinds of IO depending on etiology & \\
Mechanical IO & 236 & 97.5 \\
Paralytic ileus & 6 & 2.5 \\
Total & 242 & 100.0 \\
Types of IO depending on bowel involvement & \\
SBO & 155 & 64.0 \\
LBO & 87 & 36.0 \\
Total & 242 & 100.0 \\
Previous history of abdominal operation & \\
No & 211 & 87.1 \\
Yes & 33 & 13.6 \\
Total & 242 & 100.0 \\
Previous history of Adhesion & & \\
No & 212 & 87.6 \\
Yes & 30 & 12.4 \\
Total & 242 & 100.0 \\
Previous history of appendectomy & 100.0 \\
No & 239 & 98.8 \\
Yes & 3 & 1.2 \\
Total & 242 & \\
\hline
\end{tabular}




\section{Etiology, intra-operative findings and procedures completed}

In this study, most small bowel obstruction was found to be secondary to intussusceptions (in $30.9 \%$ of the cases) or volvulus (in $30.3 \%$ of the cases). Large bowel obstruction was mainly caused by sigmoid volvulus $(69.0 \%)$ and colonic tumor $(5.3 \%)$. As expected, the main intra-operative finding was intussusceptions, which accounted for $21 \%$ followed by adhesion and bands in $18.4 \%$ (Table 3 ). The most common intra-operative procedure was resection and anastomosis, which accounted for $40.5 \%$, followed by manual reduction and adhesion release, each accounting for $17.4 \%$ (Table 2).

Table 2 Causes of intestinal obstruction and intra-operative finding in Adam hospital medical college, February, 2014

\begin{tabular}{|c|c|c|}
\hline Variables & Frequency & Percent \\
\hline \multicolumn{3}{|l|}{ Causes of Small bowel obstruction } \\
\hline Intussusceptions & 48 & 30.9 \\
\hline Small bowel volvulus & 47 & 30.3 \\
\hline Adhesion & 42 & 27.1 \\
\hline Hernia & 9 & 5.8 \\
\hline \|liosigmoidal knotting & 5 & 4.3 \\
\hline Appendicitis & 2 & 1.3 \\
\hline Intestinal TB & 1 & 0.6 \\
\hline Mickel's diverticulitis & 1 & 0.6 \\
\hline Total & 155 & 100.0 \\
\hline \multicolumn{3}{|l|}{ Causes of large bowel obstruction } \\
\hline Sigmoid volvulus & 60 & 69.0 \\
\hline Colonic tumor & 12 & 13.8 \\
\hline Intussusceptions & 8 & 9.2 \\
\hline \|liosigmoidal knotting & 5 & 5.7 \\
\hline Fecal impaction & 2 & 2.3 \\
\hline Total & 87 & 100.0 \\
\hline \multicolumn{3}{|l|}{ Intra-operative finding } \\
\hline Intussusceptions & 48 & 21.0 \\
\hline Viable sigmoid volvulus & 42 & 18.4 \\
\hline Adhesion \& bands & 42 & 18.4 \\
\hline Viable small bowel volvulus & 31 & 13.6 \\
\hline Gangrenous SBV & 18 & 7.9 \\
\hline Gangrenous sigmoid volvulus & 18 & 7.9 \\
\hline Colonic cancer & 12 & 5.3 \\
\hline \|liosigmoidal knotting & 5 & 2.2 \\
\hline Intestinal TB & 4 & 1.7 \\
\hline Meckel's diverticulitis & 3 & 1.3 \\
\hline Others & 5 & 2.2 \\
\hline Total & 228 & 100.0 \\
\hline
\end{tabular}

\section{Management outcome of intestinal obstruction}

Nearly $94.2 \%$ of IO cases were managed by surgical procedure, whereas simple conservative management alone (i.e., naso-gastric tube insertion, intravenous antibiotics and intravenous fluid resuscitation) were applied in $5.8 \%$ of cases. Males in $65.8 \%$ of the cases and females in $20.2 \%$ were managed by operation. Of the patients that underwent laparatomy, 56 patients $(24.6 \%)$ developed an unfavorable outcome. Among these, 22 patients (39.3\%) developed wound infection, 10 (17.8\%) had facial dehiscence, 7 (12.5\%) had anastomotic leakage,5 (8.9 \%) developed septic shock and 6 (10.7\%) developed other complications like pelvic collection or pneumonia (Fig. 1).

The minimum duration of illness before arrival was $2 \mathrm{~h}$ and the maximum was $78 \mathrm{~h}$ (mean $28.63 \mathrm{~h}$; SD \pm 17.66). One hundred nineteen patients (49.2\%) presented within $24 \mathrm{~h}$, whereas $50.8 \%$ presented after $48 \mathrm{~h}$. The minimum duration of hospital stay was 2 days and the maximum duration was 30 days with an average of 9.54 days $(\mathrm{SD} \pm 4.51)$. About 120 patients (49.6\%) stayed in hospital for between 1 and 7 days, whereas 122 patients $(50.4 \%)$ stayed more than 7 days. Out of the 242 patients with IO, 234 (96.7 \%) improved and were discharged; 6 (2.5\%) died.

\section{Predictors of management outcome of intestinal obstruction}

Variables with a $p$-value of less than 0.025 in binary logistic regression analysis were entered in multivariate logistic regression to isolate the independent predictor of management outcome of IO. This allowed us to control for all variables that may be potential confounding factors. The result showed that duration of illness before surgical intervention had a significant statistical association with the management outcome of patients. Patients who presented within $24 \mathrm{~h}$ of symptom presentation are less likely to develop an unfavorable outcome, compared with patients who presented after $24 \mathrm{~h}$ (adjusted odds ratio $(\mathrm{AOR})=0.49,95 \% \mathrm{CI}: 0.25-0.97, p$-value $=0.01$ )

Patients with viable small bowel volvulus and viable sigmoid volvulus were less likely to develop an unfavorable outcome compared with patients with gangrenous small bowel volvulus $((\mathrm{AOR}=0.08,95 \% \mathrm{CI}: 0.01-0.95$, $p$-value $=0.04)$ and $(\mathrm{AOR}=0.17,95 \% \mathrm{CI}: 0.03-0.88$, $p$-value $=0.03)$, respectively) .

Bowel resection and anastomosis showed a three folds risk of developing unfavorable outcome compared with patients without resection and anastomosis of bowel $(\mathrm{AOR}=3.05, \quad 95 \%$ CI: 1.04-8.94, $p$-value $=0.02)$. Adhesion release also had a significant statistical association with management outcome $(\mathrm{AOR}=0.09,95 \% \mathrm{CI}$ : $0.01-0.69, p$-value $=0.02$ ). Intra-operative manual reduction and length of hospital stay had a significant 


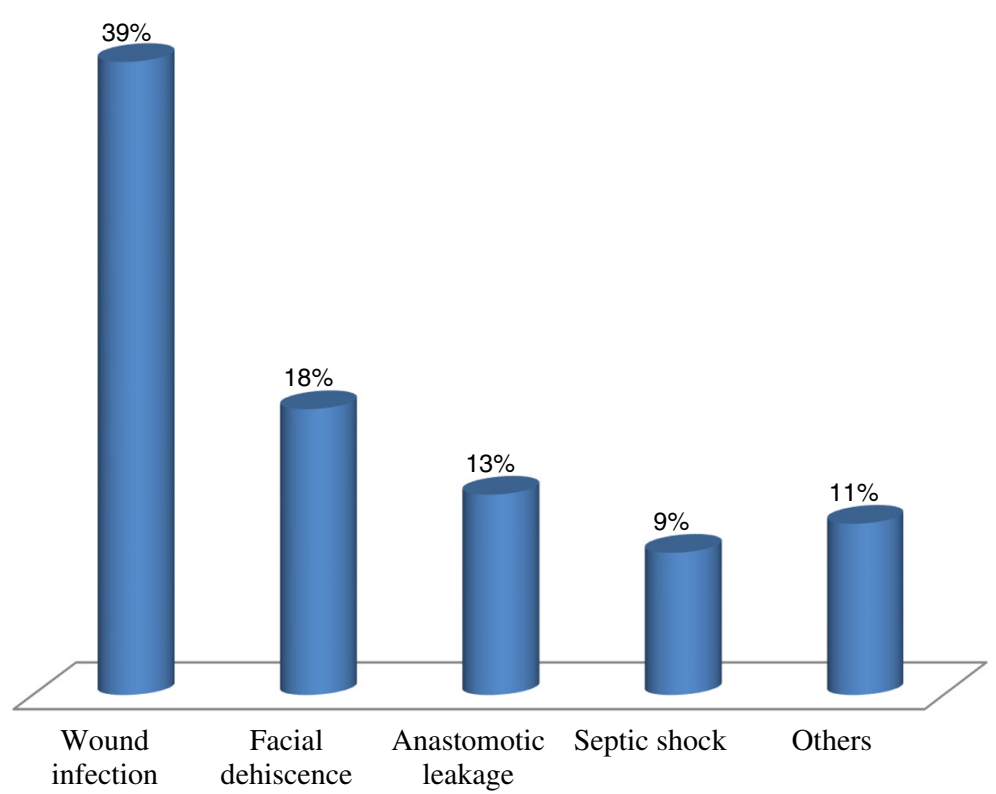

Fig. 1 Prevalence of postoperative complication of intestinal obstruction patients in Adama hospital medical college February, 2014

statistical association with management outcome $(\mathrm{AOR}=$ $0.15,95 \%$ CI: $0.03-0.68, p$-value $=0.01)$ and $(\mathrm{AOR}=0.05$, $95 \%$ CI: $0.01-0.16, p$-value $=0.001)$ respectively. Patients who had stayed for less than 7 days were less likely to develop an unfavorable outcome compared with patients who stayed for more than 7 days $(\mathrm{AOR}=0.05,95 \% \mathrm{CI}$ : $0.01-0.16, p$-value $=0.001)($ Table 3$)$.

\section{Discussion}

This study revealed the prevalence of IO to be $4.8 \%$ among all surgical patients admitted to surgical ward and $21.8 \%$ among patients admitted with a diagnosis of acute abdomen with subsequent surgical management. Indeed, world wide it has been estimated that $1 \%$ of all hospitalizations, $3 \%$ of emergency surgical admissions to general hospitals and $4 \%$ of major colostomies are secondary to IO. This study confirmed previous findings that between 12 and $17 \%$ of patients are admitted for small bowel obstruction within two years of their index operation, while approximately $3 \%$ require an operation to treat an established small bowel obstruction [10]. Currently, many patients that present to general surgery services with acute abdomen conditions are thought to have IO [8]. While IO is rare in the USA and Western Europe, it is common in Pakistan and other tropical countries. It is the leading cause of acute abdomen complaints in several African countries, including Ethiopia $[1,6,7]$. In general, there are wide variations in the prevalence of IO throughout the world depending on ethnicity, age group, dietary habits, and geographic location, among other factors. It varies from country to country and area to area in the same country [11].
Intra-operative finding like, viable small bowel volvulus (SBV) and viable sigmoid volvulus were found to be statistically significant with management outcomes. Patients with viable SBV or viable sigmoid volvulus are less likely to develop an unfavorable outcome as compared with patients with gangrenous small bowel volvulus which is similar with other studies [12, 13].

Similar studies also reported that small bowel obstruction was the most common type of IO whereas large bowel obstruction was relatively less common. Some other studies revealed that small bowel obstruction has taken the lion share and various studies agreed for the fact that small bowel obstruction is more prevalent than large bowel obstruction $[8,14]$. This fact may help us to confirm that socio-economic factors and diet might be responsible for the causes of this problem in some developing countries. A previous study in an Ethiopian hospital found that small bowel and large bowel obstruction account for 52.3 and $46.7 \%$ respectively [7]. Mechanical IO were most common obstruction in our study, whereas adynamic obstruction was less prevalent. A study in Pakistan also confirmed that paralytic ileus was common and causes of small bowel obstruction were mostly due to intussusceptions and volvulus [1].

Intra-operative procedures like bowel resection and anastomosis have significant statistical association with management outcome. Bowel resection and anastomosis has a three times high risk of developing an unfavorable outcome compared with patients without resection and anastomosis of bowel.

Adhesion release and manual reduction intraoperatively also demonstrated a significant statistical 
Table 3 Factors associated with management outcome of intestinal obstruction in Adama hospital medical college, February, 2014

\begin{tabular}{|c|c|c|c|c|c|}
\hline \multirow[t]{2}{*}{ Variables } & \multicolumn{2}{|c|}{ Management outcomes } & \multirow[t]{2}{*}{ COR $(95 \% \mathrm{Cl})$} & \multirow[t]{2}{*}{ AOR $(95 \% \mathrm{Cl})$} & \multirow[t]{2}{*}{$P$-value } \\
\hline & Favorable [N] & Unfavorable [N] & & & \\
\hline \multicolumn{6}{|l|}{ Age } \\
\hline$<14$ years & 52 & 8 & $0.287(0.11-0.76)$ & $0.99(0.11-2.13)$ & 0.998 \\
\hline $14-24$ years & 21 & 7 & $0.62(0.21-1.79)$ & $0.59(0.109-3.21)$ & 0.543 \\
\hline $25-34$ years & 32 & 12 & $0.70(0.28-1.74)$ & $0.86(0.23-3.17)$ & 0.828 \\
\hline $35-44$ years & 27 & 5 & $0.34(0.11-1.08)$ & $0.35(0.08-1.48)$ & 0.158 \\
\hline $45-55$ years & 45 & 9 & $0.64(0.242-1.73)$ & $0.28(0.07-1.08)$ & 0.066 \\
\hline$>55$ years & 28 & 15 & 1 & 1 & \\
\hline \multicolumn{6}{|l|}{ Occupation } \\
\hline Farmer & 64 & 26 & 1 & 1 & \\
\hline Merchant & 18 & 7 & $0.95(0.35-2.56)$ & $1.93(0.56-6.56)$ & 0.291 \\
\hline House wife & 14 & 13 & $2.28(0.94-5.52)$ & $3.28(0.97-11.11)$ & 0.056 \\
\hline Student & 18 & 1 & $0.14(0.02-1.07)$ & $0.97(1.87-2.61)$ & 0.998 \\
\hline Governmental employer & 23 & 2 & $0.21(0.05-0.97)$ & $0.14(0.02-1.09)$ & 0.061 \\
\hline \multicolumn{6}{|l|}{ Duration of illness } \\
\hline$\leq 24 \mathrm{~h}$ & 101 & 18 & $0.39(0.21-0.74)$ & $0.49(0.25-0.97)$ & $0.014^{*}$ \\
\hline$>24 h$ & 85 & 38 & 1 & 1 & \\
\hline \multicolumn{6}{|l|}{ Intra-operative finding } \\
\hline Gangrenous SBV & 9 & 9 & 1 & 1 & \\
\hline Gangrenous SV & 8 & 10 & $1.25(0.33-4.63)$ & $1.95(0.34-10.95)$ & 0.447 \\
\hline Adhesion \& bands & 28 & 14 & $0.50(0.16-1.54)$ & $5.90(0.72-4.80)$ & 0.098 \\
\hline Viable SBV & 29 & 2 & $0.07(0.01-0.37)$ & $0.08(0.01-0.95)$ & $0.046^{*}$ \\
\hline Viable SV & 34 & 7 & $0.21(0.06-0.71)$ & $0.17(0.03-0.88)$ & $0.035^{*}$ \\
\hline Intussusceptions & 49 & 7 & $0.14(0.04-0.48)$ & $0.49(0.07-3.22)$ & 0.460 \\
\hline Colonic Cancer & 8 & 4 & $0.50(0.11-2.27)$ & $0.27(0.04-1.64)$ & 0.157 \\
\hline Hernia & 8 & 1 & $0.12(0.01-1.216)$ & $0.12(0.01-1.65)$ & 0.114 \\
\hline Illiosigmoidal knot & 4 & 1 & $0.25(0.023-2.69)$ & $1.40(0.04-43.13)$ & 0.844 \\
\hline \multicolumn{6}{|c|}{ Intra-operative procedure done } \\
\hline \multicolumn{6}{|l|}{ Resection\& Anastomosis } \\
\hline Yes & 63 & 35 & $3.02(1.61-5.69)$ & $3.05(1.04-8.94)$ & $0.041^{*}$ \\
\hline No & 109 & 21 & 1 & 1 & \\
\hline \multicolumn{6}{|l|}{ Adhesion release } \\
\hline Yes & 33 & 9 & $0.83(0.37-1.86)$ & $0.09(0.01-0.69)$ & $0.020^{*}$ \\
\hline No & 139 & 47 & 1 & 1 & \\
\hline \multicolumn{6}{|l|}{ Manual Reduction } \\
\hline Yes & 41 & 2 & $0.12(0.02-0.52)$ & $0.15(0.03-0.68)$ & $0.014^{*}$ \\
\hline No & 131 & 54 & 1 & 1 & \\
\hline \multicolumn{6}{|l|}{ Hospital stay } \\
\hline$\leq 7$ days & 117 & 3 & $0.03(0.01-0.11)$ & $0.05(0.01-0.16)$ & $0.001^{*}$ \\
\hline$>7$ days & 69 & 53 & 1 & 1 & \\
\hline
\end{tabular}

*Significant at $p$-value $<0.051$ is reference

$S B V$ small bowel volvulus, SV sigmoid volvulus, SBO small bowel obstruction, 10 intestinal obstruction

association with management outcome. According to this study, the common causes of intestinal obstruction were mostly due to adhesion whereas obstructed/strangulated hernia was less prevalent. A study conducted in Western Sudan, showed obstructed/strangulated hernia to be more prevalent than adhesion, while SBV was found to be the 
least prevalent by far [15]. Similarly, a study conducted in Uganda also confirmed that the four leading causes of obstruction were hernias, adhesions, volvulus and intussusceptions [16]. A six years study of 56 cases of IO seen and treated at Asir Central Hospital in Saudi Arabia found that adhesion was the most common cause of IO, however, hernias were a leading cause of obstruction in most African centers. This might imply that the culture, food, age of the patients and geography have huge influences on the prevalence of obstruction. A study also confirmed that IO is almost certainly affected by the paucity of surgical services in the region [17]. Intussusception was the most common IO in our study, especially in children, indicates a possible association with upper respiratory tract infections. Patients' large bowels were obstructed secondary to sigmoid volvulus in $69.0 \%$ of the cases. This was not surprising, as sigmoid volvulus has been reported to be a frequent cause of IO in a African countries [17]. A study reported that sigmoid volvulus was the leading cause of IO in the northern part of Ethiopia [7]. Sigmoid volvulus is also the leading cause of large bowel obstruction in most of the sub-Saharan region $[9,18]$.

Duration of illness before surgical intervention has significant statistical association with management outcome of patients. Patients who presented within $24 \mathrm{~h}$ duration of illness are less likely to develop unfavorable outcome compared with patients who presented after 24 h. A study in Rwanda indicated that more than threefourths of patients presented after $24 \mathrm{~h}$ [19], however, in our study half of the patients presented within $24 \mathrm{~h}$. Delayed presentation and/or surgical intervention frequently results in relatively poor surgical outcomes and/or longer hospital stays [17].

Our study also revealed that patients who stayed for less than 7 days were less likely to develop unfavorable outcomes compared with patients who stayed for more than 7 days. By comparison, one study of pattern of acute abdomen in Butajira, Ethiopia showed that the average hospital stay was 9 days and a third of patients developed one or more acute complications $[9,20]$. The difference in IO outcome may be associated with late duration of patients' illness to hospital due to lack of awareness about the burden and impacts of the problem. The potential reasons for lower mortality rate in our study may be due to early intervention of the obstruction before complications occur and adequate preoperative resuscitation which might be expected to decrease mortality.

As to the management, laparotomy or surgical incision was the most common method of IO management in this investigation; our results indicated slightly higher rates than previous research conducted in Nigeria and USA [21, 22]. The outcome of laparotomy might be affected by different factors, such as cause of obstruction, duration of illness, age, presence of peritonitis and complication detection time.

This study was subject to certain potential limitations. The use of secondary data confers lack of oversight about how the original data were collected. For instance, some patient information may have been missed or incorrectly recorded. This in turn may lead to under or over estimations of the finding. As much as possible, we tried to manage and minimize these limitations through careful selection of records of the patients.

\section{Conclusions}

In conclusion, small bowel obstruction was more prevalent than large bowel obstruction in this study. Intussusceptions and sigmoid volvulus were the leading causes of small and large bowel obstruction, respectively. Laparotomy was the most common means of IO management, while bowel resection and anastomosis was the most common intra operative procedure. The most commonly encountered postoperative complications were wound infection followed by facial dehiscence.

Our findings suggest that health professionals in the hospital and district should increase public awareness on IO by providing appropriate health information. Physicians should diagnose intestinal early and appropriate interventions should be taken on time before the intestine develops gangrene. Wound infection in the study area should be improved because it is the most common postoperative complication in the area. This can be decreased by appropriate surgical technique and wound care with sterile techniques. Card room staff should improve record keeping in the hospital because some handwriting was not readable and some charts were lost. Further research using prospective study design is warranted as a way to overcome the limitations of secondary data in the current retrospective research that preclude generalization to the whole population.

\section{Abbreviations \\ AOR, adjusted odds ratio; $\mathrm{Cl}$, confidence interval; $\mathrm{COR}$, crude odds ratio; IO, intestinal obstruction; $\mathrm{LBO}$, large bowel obstruction; $\mathrm{MPH}$, Master of Public Health; SBO, small bowel obstruction; SBV, small bowel volvulus; SD, standard deviation; SPSS, Statistical Packages for Social Science; WHO, World Health Organization}

\section{Acknowledgement}

We would like to acknowledge the assistance of the Jimma University, College of Public Health and Medical Sciences in undertaking this research. We would also like to express our gratitude to officials and staff working in Adama Hospital Medical College; the educational office at this institution, study participants, data collectors and supervisors. Last but not least, we would like to thank Nicole Bergen from University of Ottawa in Canada for her valuable help in editing the English language of the manuscript.

\section{Funding}

This research was funded by Jimma University, College of Public Health and Medical Sciences. 


\section{Availability of data and materials}

The datasets supporting the conclusions of this article are included within the article.

\section{Authors' contributions}

UN contributed to the conceptualization, conducted the literature review, design, analysis and the writing of the paper. DH, NF and AM contributed on research design, analysis, the conceptualization and writing the manuscript. All authors read and approved the final submitted paper.

\section{Competing interests}

The authors declare that they have no competing interests.

\section{Ethics approval and consent to participate}

The institutional review board of Jimma University College of Public Health and Medical Sciences approved the study. A formal letter of support was written from College IRB to Adama Hospital administrator's office, and the procedures were in compliance with Helsinki Declaration.

\section{Author details}

${ }^{1}$ Department of obstetrics/gynecology and surgery coordinating office, Jimma University specialized hospital, Jimma, Ethiopia. ${ }^{2}$ Department of Health Education and Behavioral Sciences, Jimma University, Jimma, Ethiopia. ${ }^{3}$ Department of Epidemiology, Jimma University, Jimma, Ethiopia.

${ }^{4}$ Department of Health Education and Behavioral Sciences, College of Health Sciences, Jimma University, PO Box 378, Jimma, Ethiopia.

\section{Received: 30 October 2015 Accepted: 19 May 2016}

\section{Published online: 04 June 2016}

\section{References}

1. Ullah S, Khan M. Intestinal obstruction: A Spectrum of causes, Department of Surgery, Postgraduate Medical Institute Lady Reading Hospital, Peshawar Pakistan. JPMI. 2008;8(1):210-3.

2. Cirocchi R, Abraha I, Farinella E, Montedori A, Sciannameo F. Laparoscopic versus open surgery in small bowel obstruction. Cochrane Database Syst Rev. 2010:17(2):751-5.

3. Shaikh MS, Dholia KR. Current Spectrumof Acute Intestinalobstructionat CMC Larkana. Medical Channel. 2010;16(2):74-8.

4. Winslet MC, William NS, Bulstrode CJK, O'Connel PR. Bailey and love's short practice of surgery: intestinal obstruction. 25th ed. London: Arnold; 2008. p. 1189-202.

5. Quill DS, Devlin HB, Deham KR. Surgical operation rates. A 12 year experiences in Stockton on tees. Ann R Coll Surg Engl. 2007:65(7):248-53.

6. Khanzada TW, Samad A. Etiological spectrum of dynamic intestinal obstruction, Department of Surgery, Isra University Hospital, Hyderabad, Pakistan. Gomal J Med Sci. 2006;12(1):35-6.

7. Adesunkanmi ARK, Agbakwuru E. Changing pattern of acute intestinal obstruction in a tropical African population. East Afr Med J. 2011;73(11):727-31.

8. Kotiso B, Abdurahman Z. Pattern of acute abdomen in adult patients in Tikur Anbessa Teaching Hospital, Addis Ababa. East Cent Afr J Surg. 2006;12(1):47-52.

9. Demissie M. Small intestinal volvulus in Southern Ethiopia. EAMJ. 2005;78(4):208-11.

10. Balouch NA, Baber KM, Mengal MA, Baber SAA. Spectrum of mechanical obstruction. JSP (Int). 2007;7(1):7-9.

11. Espinoza R, BalbontÄn P, Feuerhake S. Acute abdomen in the elderly. Rev Med Chil. 2004;132(12):1505-12.

12. Ahmed M, Mahmood TR, Ansari AS, Ahmed I, Ahmed M. Spectrum of mechanical obstruction in adult. JSP (Int). 2006;6(4):19-21.

13. Ntakiyiruta G, Mukarugwiro B. The pattern of intestinal obstruction at Kibogola Hospital, a Rural Hospital in Rwanda. East Cent Afr J Surg. 2011;16(1):65-70

14. Johson. Prevalence of sigmoid volvulus in Tikur Anbessa hospital: Addis Abeba. EMJ. 2002;4(2):129-32.

15. Doumi et al. Emergency laparotomy for acute sigmoid in El Obeid Hospital, western Sudan. JMS. 2007;2(1):54-66.

16. Macutkiewicz C, Carlson GL. Intestinal obstruction. Surg Int Uganda. 2005;70:10-4.

17. Heis HA et al. Sigmoid volvulus in the Middle East. World J Surg. 2008:32:459-64.
18. Nega B. Pattern of acute abdomen and variables associated with advert outcome in a rural primary setting. Ethiop Med J. 2009;47(2):143-51.

19. Ntakiyiruta G, Mukarugwiro B. The pattern of intestinal obstruction at Kibogola Hospital, a Rural Hospital in Rwanda. East Cent Afr J Surg. 2009;14(2):103-8.

20. Ayalew T. Small intestinal volvulus in adults of Gondar region, N, W Ethiopia. Ethiop Med J. 2001;30:111-4.

21. Osuigwe AN, Anyanwu. Acute intestinal obstruction in Nnewi Nigeria: A five year reviews. Niger J Surg Res. 2006:4(1):107-11.

22. Nemes R, Vasile I, Curca T, Paraliov T, Pasalega M, Mesina C, et al. Acute bowel obstruction- the main complication of colorectal cancer therapeutical options. Rom J Gastroenterol. 2004;13(2):109-12.

\section{Submit your next manuscript to BioMed Central and we will help you at every step:}

- We accept pre-submission inquiries

- Our selector tool helps you to find the most relevant journal

- We provide round the clock customer support

- Convenient online submission

- Thorough peer review

- Inclusion in PubMed and all major indexing services

- Maximum visibility for your research

Submit your manuscript at www.biomedcentral.com/submit 